\title{
Diffuse Large B-Cell Lymphoma: Optimizing Therapy for Relapsed/Refractory Disease
}

\author{
Presented by Matthew J. Matasar, MD
}

\section{ABSTRACT}

Despite a growing therapeutic arsenal to treat relapsed/refractory (R/R) diffuse large B-cell lymphoma (DLBCL), outcomes remain poor. Only approximately half of patients with this disease are eligible to receive curative autologous stem cell transplant, and of those, only half will be cured. Moreover, for patients who are transplant-ineligible, there are few effective options. Dr. Matthew J. Matasar discussed the current unmet needs in the treatment of patients with R/R DLBCL, treatment strategies in the second- and third-line settings, and emerging therapeutic options.

J Natl Compr Canc Netw 2021;19(11.5):1327-1330 doi: 10.6004/jnccn.2021.5109

With only approximately half of patients eligible to receive curative autologous stem cell transplant (ASCT), outcomes remain poor for patients with relapsed/refractory $(\mathrm{R} / \mathrm{R})$ diffuse large B-cell lymphoma (DLBCL). At the NCCN 2021 Virtual Congress: Hematologic Malignancies, Matthew J. Matasar, MD, Associate Member, Lymphoma Service, and Regional Care Network Medical Site Director, Memorial Sloan Kettering Bergen, New Jersey, discussed current unmet needs for patients with R/R DLBCL, second- and third-line therapeutic strategies, and emerging therapeutics. "It's clear to those of us who practice in the field that there remains a significant unmet need in the treatment of patients with R/R DLBCL," said Dr. Matasar. "For patients who are ineligible for ASCT, there are relatively few effective options and no clearly defined standards of care, and the prognosis can be quite poor."

According to data from the SCHOLAR- 1 study, ${ }^{1}$ which was a patient-level pooled retrospective analysis of both phase III and observational studies of patients with $R / R$ DLBCL, the overall response rate (ORR) to chemotherapy is $26 \%$ and the complete response (CR) rate is $7 \%$.

\section{Heterogeneous Practice Patterns}

As Dr. Matasar detailed, community-based treatment of R/R DLBCL in the United States remains heterogeneous. A retrospective cohort study of data from Flatiron Health of patients with DLBCL diagnosed between 2011 and 2018 showed that rituximab + ifosfamide/carboplatin/ etoposide (R-ICE) is the most used treatment regimen in the second-line setting (23\%), followed by bendamustine + rituximab (BR; 8\%), rituximab + cyclophosphamide/ doxorubicin/vincristine/prednisone (R-CHOP; 7\%), rituximab + gemcitabine/oxaliplatin (R-GemOx; $6 \%$ ), and several other regimens. ${ }^{2}$ In the third-line setting, R-GemOx remains the most used treatment regimen (15\%). According to Dr. Matasar, however, these data predate the commercial availability of CAR T-cell therapy. "We hope and expect to see a change in practice patterns moving forward," he said.

\section{Transplant-Eligible Versus Transplant-Ineligible Patients}

For second-line R/R DLBCL, patients are stratified by ASCT eligibility. Those who are eligible receive platinumbased chemotherapy with R-ICE or R-DHAX (dexamethasone/cytarabine/oxaliplatin), and those who experience a partial response or better then receive consolidative treatment with ASCT (Figure 1).

Although it is standard to use platinum-based chemotherapy in the second-line setting, no individual regimen has been shown to be superior to others (Table 1). ${ }^{3-5}$ "ORRs preceding transplant seem to be quite comparable, regardless of the platinum-based regimen used," said Dr. Matasar. "It is known, however, that outcomes with platinum-based chemotherapy remain poor in patients who received rituximabbased chemoimmunotherapy in the first-line setting."

Conversely, patients who are ineligible for ASCT under the current paradigm are stratified by eligibility for CAR T-cell therapy. Those who are eligible are treated with regimens "least likely to interfere with subsequent CAR T-cell therapy," said Dr. Matasar. Those considered 




Figure 1. DLBCL second-line approach.

Abbreviations: $3 \mathrm{~L}$, third line; BEAM, etoposide/cytarabine/melphalan; BR, bendamustine/rituximab; DLBCL, diffuse large B-cell lymphoma; HDT/ASCR, high-dose therapy with autologous stem cell rescue; non-GCB, non-germinal center B-cell like; pola, polatuzumab; R/R, relapsed/refractory; R-GemOx, rituximab + gemcitabine/ oxaliplatin; RDHAX, rituximab/dexamethasone/cytarabine/oxaliplatin; RICE, rituximab/ifosfamide/carboplatin/etoposide; Tafa + len, tafasitamab + lenalidomide.

ineligible for both ASCT and cellular therapy, on the other hand, can receive anti-CD19-directed therapy, such as $\mathrm{BR}+$ polatuzumab or tafasitamab + lenalidomide.

\section{Polatuzumab Vedotin}

According to Dr. Matasar, BR or R-GemOx were traditionally used in transplant-ineligible patients with R/R DLBCL, but real-world evidence showed disappointing outcomes. Polatuzumab vedotin-piiq, an antibody-drug conjugate, was established as the new standard for transplant-ineligible patients based on results of the GO29365 study, which randomly assigned 80 patients to either BR or BR + polatuzumab vedotin. ${ }^{6}$ Results of this study showed an overall survival improvement from 4.7 months with BR alone to $>1$ year with the addition of polatuzumab vedotin. The combination was adequately tolerated, said Dr. Matasar, who noted an increase in both myelosuppression and neuropathy with the polatuzumab vedotin combination, but there were no new safety signals.

Polatuzumab vedotin is also currently being studied in combination with R-ICE in transplant-eligible patients to improve ORRs and the ability to deliver ASCT to more patients.

\section{Tafasitamab + Lenalidomide}

Polatuzumab vedotin is not the only new addition to the therapeutic armamentarium. Data from the L-MIND trial, ${ }^{7}$ which evaluated combination lenalidomide +

\section{Table 1. Salvage Regimens in R/R DLBCL}

\begin{tabular}{|c|c|c|c|c|c|c|}
\hline Study & Salvage Regimens & ORR & $\begin{array}{l}\text { Received } \\
\text { ASCT }\end{array}$ & $P$ Value & OS Rate & $P$ Value \\
\hline $\begin{array}{l}\text { CORAL }^{3, a} \\
\mathrm{~N}=396\end{array}$ & $\begin{array}{l}\text { R-DHAP } \\
\text { vs R-ICE }\end{array}$ & $\begin{array}{l}63 \% \\
64 \%\end{array}$ & $\begin{array}{l}54 \% \\
50 \%\end{array}$ & .6 & $\begin{array}{l}3 \text {-year: } 51 \% \\
\text { 3-year: } 47 \%\end{array}$ & .4 \\
\hline $\begin{array}{l}\mathrm{NCIC}-\mathrm{CTG}^{4, b} \\
\mathrm{~N}=619\end{array}$ & $\begin{array}{l}\text { R-DHAP } \\
\text { vs R-GDP }\end{array}$ & $\begin{array}{l}44 \% \\
45 \%\end{array}$ & $\begin{array}{l}49 \% \\
52 \%\end{array}$ & .95 & $\begin{array}{l}\text { 4-year: } 39 \% \\
\text { 4-year: } 39 \%\end{array}$ & .78 \\
\hline $\begin{array}{l}\text { ORCHARRD }^{5} \\
\mathrm{~N}=445\end{array}$ & $\begin{array}{l}\text { R-DHAP } \\
\text { vs O-DHAP }\end{array}$ & $\begin{array}{l}94 \% \\
84 \%\end{array}$ & $\begin{array}{l}37 \% \\
33 \%\end{array}$ & .41 & $\begin{array}{l}\text { 2-year: } 38 \% \\
\text { 2-year: } 41 \%\end{array}$ & .38 \\
\hline
\end{tabular}

Abbreviations: ASCT, autologous stem cell transplantation; DLBCL, diffuse large B-cell lymphoma; ODHAP, ofatumumab/dexamethasone/cytarabine/cisplatin; ORR, overall response rate; OS, overall survival; R-DHAP, rituximab/dexamethasone/cisplatin/cytarabine; R-GDP, rituximab/gemcitabine/dexamethasone/ cisplatin; R-ICE, rituximab/ifosfamide/etoposide/carboplatin; R/R, relapsed/refractory.

${ }^{a}$ ClinicalTrials.gov identifier: NCT00137995.

${ }^{b}$ ClinicalTrials.gov identifier: NCT00078949; included transformed and T-cell lymphoma. 
tafasitamab-an anti-CD19-directed monoclonal antibody - in patients with relapsed DLBCL showed an ORR of $60 \%$ by investigator assessment, with a CR rate of $43 \%$. Median progression-free survival was 12 months. However, among responders, the median duration of response improved to 22 months, said Dr. Matasar. Furthermore, for patients who achieved a CR, the median duration of response has not been reached.

"People who achieve a CR can enjoy a very durable response with the L-MIND regimen," said Dr. Matasar, who noted that median overall survival has not been reached either.

\section{CAR T-Cell Therapy: Third-Line Setting and Beyond}

There are currently 3 CAR T-cell therapies that are FDAapproved for multiply relapsed DLBCL: axicabtagene ciloleucel, tisagenlecleucel, and lisocabtagene maraleucel (Table 2).$^{8-10}$ As Dr. Matasar reported, all 3 agents have demonstrated very high ORRs and CR rates, but there are differences in terms of toxicity and eligible patient populations in the underlying studies. "The rates of CRS [cytokine-release syndrome] requiring the use of tocilizumab or steroids, the overall severity of CRS, and the risk of neurotoxicity differ among the CAR T-cell agents currently available," said Dr. Matasar. "Our team-based approach evaluates both the comparative efficacy and toxicity of these agents to assign the best choice to each individual patient."

According to Dr. Matasar, data from Memorial Sloan Kettering Cancer Center have demonstrated superior rates of CR, 12-month progression-free survival, and overall survival for patients receiving CAR T-cell therapy in the third-line setting and beyond compared with the use of other agents. ${ }^{11}$

\section{Loncastuximab Tesirine}

Loncastuximab tesirine-lpyl, a CD19-directed antibody-drug conjugate, is another FDA-approved option in the treatment of R/R DLBCL in the third line and beyond. Approval of loncastuximab tesirine was based on data from the LOTIS-2 trial, ${ }^{12}$ which demonstrated approximately a $50 \%$ response in all patients, including those with DLBCL not otherwise specified, and a median duration of response of approximately 10 months. Long-term toxicities of note included myelosuppression and a high rate of gamma-glutamyltransferase elevation.

\section{Emerging Therapies}

Dr. Matasar also reported several emerging therapies that are not currently FDA-approved, including bispecific antibodies, which are antibodies that are bivalent (not univalent) and directed against B-cell antigens such as CD20 and CD3. Mosunetuzumab, glofitamab, epcoritamab, and IGM-2323 are bispecific antibodies in ongoing development.

According to Dr. Matasar, GO29781, which is evaluating intravenous mosunetuzumab in the treatment of $\mathrm{R} / \mathrm{R}$ B-cell lymphomas, both indolent and aggressive, is the study that is furthest along. ${ }^{13}$ Despite very-high-risk patient characteristics, Dr. Matasar called the rates of ORR and CR "relatively encouraging." ORRs are approximately 35\%, and CR rates are between $20 \%$ and $25 \%$, depending on patient characteristics, he said. Further, he noted that mosunetuzumab is being developed for subcutaneous administration. "Suffice it to say, the entire field is moving very quickly, and we are very much encouraged by the ongoing development of bispecific antibodies in this disease state."

Anti-CD47 antibodies, including magrolimab, TTI-621, and TTI-622, are also in ongoing clinical development along

\section{Table 2. CAR T-Cell Therapy in Multiply Relapsed DLBCL}

\begin{tabular}{|c|c|c|c|}
\hline & KTE-C19 ${ }^{8}$ & CTL0199 & $\mathrm{JCAR} 17^{10}$ \\
\hline Drug name & Axicabtagene ciloleucel & Tisagenlecleucel-T & Lisocabtagene maraleucel \\
\hline $\begin{array}{l}\text { Clinical trial } \\
\quad \text { (ClinicalTrials.gov identifier) }\end{array}$ & $\begin{array}{l}\text { ZUMA-1 } \\
\text { (NCT02348216) }\end{array}$ & $\begin{array}{l}\text { JULIET } \\
\text { (NCT02445248) }\end{array}$ & $\begin{array}{l}\text { TRANSCEND NHL } 001 \\
\text { (NCT02631044) }\end{array}$ \\
\hline Phase & Phase I/II & Phase Ila & Phase I \\
\hline Dose level & $2 \times 10^{6}$ cells $/ \mathrm{kg}$ & $5 \times 10^{8}$ cells & $5 \times 10^{7}$ cells \\
\hline Conditioning chemotherapy & Low-dose Cy/Flu x $3 \mathrm{~d}$ & $\begin{array}{l}\text { Variety; based on clinical } \\
\text { features and past therapies }\end{array}$ & Low-dose Cy/Flu x $3 \mathrm{~d}$ \\
\hline Evaluable patients & $\begin{array}{l}\text { DLBCL }(n=77) \\
\text { tFL/PMBCL }(n=24)\end{array}$ & $\operatorname{DLBCL}(n=51)$ & $\begin{array}{l}\text { DLBCL }(n=40) \\
\text { Transformed DLBCL }(n=14) \\
\text { FL grade 3B }(n=1)\end{array}$ \\
\hline Response rates & $\begin{array}{l}\text { ORR, } 82 \% \\
\text { CR, } 54 \%\end{array}$ & $\begin{array}{l}\text { ORR, } 59 \% \\
\text { CR, } 43 \%\end{array}$ & $\begin{array}{l}\text { ORR, } 76 \% \\
\text { CR, } 52 \%\end{array}$ \\
\hline
\end{tabular}

Abbreviations: CR, complete response; Cy, cyclophosphamide; DLBCL, diffuse large B-cell lymphoma; FL, follicular lymphoma; Flu, fludarabine; ORR, overall response rate; $\mathrm{PMBCL}$, primary mediastinal large $\mathrm{B}$-cell lymphoma; $\mathrm{tFL}$, transformed follicular lymphoma. 
with the development of checkpoint inhibitor therapy. "Checkpoint monotherapy has not been proven to be highly active in the treatment of R/R DLBCL, but there is a lot of work ongoing, including the combination of anti-LAG-3 with anti-PD-1 therapy in an attempt to enhance the activity of checkpoint inhibition in either CAR T-cell-naïve or CAR T-cell-experienced patients, and we await ongoing data from several studies," said Dr. Matasar.

Disclosures: Dr. Matasar has disclosed receiving consulting fees from Bayer HealthCare, Daiichi-Sankyo Co., Genentech, Inc., Juno Therapeutics, Inc.,
Merck \& Co., Inc., Roche Laboratories, Inc., Rocket Medical, Seattle Genetics, Inc., Takeda Pharmaceuticals North America, Inc., and Teva Pharmaceutical Industries Ltd.; receiving grant/research support from Bayer HealthCare, Genentech, Inc., GlaxoSmithKline, IGM Biosciences, Immunovaccine Technologies, Janssen Pharmaceutica Products, LP, Pharmacyclics, Roche Laboratories, Inc., Rocket Medical, and Seattle Genetics, Inc.; receiving honoraria from Bayer HealthCare, Genentech, Inc., GlaxoSmithKline, Immunovaccine Technologies, Janssen Pharmaceutica Products, LP, Pharmacyclics, Roche Laboratories, Inc., Seattle Genetics, Inc., and Takeda Pharmaceuticals North America, Inc.; and having ownership/partnership/principal in Merck \& Co. Inc.

Correspondence: Matthew J. Matasar, MD, Memorial Sloan Kettering Cancer Center, 1275 York Avenue, New York, NY 10065

Email: matasarm@mskcc.org

\section{References}

1. Crump M, Neelapu SS, Farooq U, et al. Outcomes in refractory diffuse large B-cell lymphoma: results from the international SCHOLAR-1 study. Blood 2017;130:1800-1808.

2. Herrera AF, Ogbu U, Ku G, Chuo CY. Real-world bendamustine use in relapsed/refractory diffuse large B-cell lymphoma (R/R DLBCL) [abstract] Hematol Oncol 2019;37(Suppl 2):428-429. Abstract 388.

3. Gisselbrecht C, Glass B, Mounier N, et al. Salvage regimens with autologous transplantation for relapsed large B-cell lymphoma in the rituximab era. J Clin Oncol 2010;28:4184-4190.

4. Crump M, Kuruvilla J, Couban S, et al. Randomized comparison of gemcitabine, dexamethasone, and cisplatin versus dexamethasone, cytarabine, and cisplatin chemotherapy before autologous stem-cell transplantation for relapsed and refractory aggressive lymphomas: NCICCTG LY.12. J Clin Oncol 2014;32:3490-3496.

5. van Imhoff GW, McMillan A, Matasar MJ, et al. ofatumumab versus rituximab salvage chemoimmunotherapy in relapsed or refractory diffuse large B-cell lymphoma: the ORCHARRD study. J Clin Oncol 2017;35:544-551.

6. Lu T, Gibiansky L, Li X, et al. Exposure-safety and exposure-efficacy analyses of polatuzumab vedotin in patients with relapsed or refractory diffuse large B-cell lymphoma. Leuk Lymphoma 2020;61:2905-2914.

7. Salles G, Duell J, González Barca E, et al. Tafasitamab plus lenalidomide in relapsed or refractory diffuse large B-cell lymphoma (L-MIND): a multicentre, prospective, single-arm, phase 2 study. Lancet Oncol 2020; 21:978-988.

8. Locke FL, Neelapu SS, Bartlett NL, et al. Primary results from ZUMA-1: a pivotal trial of axicabtagene ciloleucel (axicel; KTE-C19) in patients with refractory aggressive non-Hodgkin lymphoma (NHL) [abstract]. Presented at the 2017 AACR Annual Meeting; April 1-5, 2017; Washington, DC. Abstract CT019.

9. Schuster SJ, Bishop MR, Tam CS, et al. Tisagenlecleucel in adult relapsed or refractory diffuse large B-cell lymphoma. N Engl J Med 2019;380:45-56.

10. Abramson JS, Palomba ML, Gordon LI, et al. CR rates in relapsed/refractory (R/R) aggressive B-NHL treated with the CD19-directed CAR T-cell product JCAR017 (TRANSCEND NHL 001) [abstract]. J Clin Oncol 2017; 35(Suppl):Abstract 7513.

11. Sermer D, Batlevi $C$, Palomba ML, et al. Outcomes in patients with DLBCL treated with commercial CAR T cells compared with alternate therapies. Blood Adv 2020;4:4669-4678.

12. Caimi PF, Ai W, Alderuccio JP, et al. Loncastuximab tesirine in relapsed or refractory diffuse large B-cell lymphoma (LOTIS-2): a multicentre, open-label, single-arm, phase 2 trial. Lancet Oncol 2021;22:790-800.

13. Matasar MJ, Cheah CY, Yoon DH, et al. Subcutaneous mosunetuzumab in relapsed or refractory B-cell lymphoma: promising safety and encouraging efficacy in dose escalation cohorts [abstract]. Blood 2020; 136(Suppl 1):45-46. Abstract 2096. 